\title{
Abstract Author Index
}

A

Abdelfattah, M., A-3

Aghassi, E., A-3

Alcozer, R., A-14

Al-Otaibi, L.M., A-19

Amaroli, A., A-3

Antonelli, R., A-14, A-20

Aprea, L., A-9

Arduino, PG., A-17

Arnabat-Domínguez, J., A-4

B

Baciut, G., A-5

Baciut, M., A-5

Barreira, E., A-14

Barylyak, A., A-4

Bellini, P., A-6, A-17, A-21

Benedicenti, S., A-3

Bertino, K., A-14

Bianchi Zamataro, C., A-22

Bisheimer, C.M., A-10

Bordea, R., A-5

Borodenko, D., A-15

Bosotti, M., A-4, A-9, A-19

Bossi, E., A-4, A-9, A-15

Bou Tayeh, R., A-10

Bran, S., A-5

Briga, C., A-14

Broccoletti, R., A-17

Broglia, S., A-15

\section{C}

Cabras, M., A-15, A-17

Cafaro, A., A-15, A-17

Campian, R.S., A-5

Candotto, V., A-4, A-15, A-19, A-20

Caprioglio, C., A-13

Caramel Juvino, A., A-22

Cassoni, A., A-3

Castro, P., A-8, A-18, A-22

Castro, P.AA., A-21

Castro, P.A.L., A-13

Celli, D., A-7

Chegeni, E., A-4

Cherepinska, A-2

Chumakova, Y., A-15

Consolo, U., A-17

Corcione, L., A-19

Crippa, R., A-5

Crisan, B., A-5

Crisan, L., A-5

\section{D}

Da Ana, P., A-22

Da Ana, P.A., A-13

Daghero, O., A-15
D'Alessandro, L., A-10, A-16, A-18, A-20

De Filippis, M., A-16

Dehghan, M.M., A-7

Del Vecchio, A., A-5, A-9, A-10, A-16, A-22

Di Gioia Cira, R.T., A-16

Dinu, C., A-5

Dogandzhiyska, V., A-16

Domínguez-Mompell, R., A-10

E

Elia, F., A-17

El Mobadder, M., A-6

España-Tost, A., A-4

F

Farzad Mohajeri, S., A-7

Fekrazad, R., A-7

Ferreira, R., A-22

Figueiredo, R., A-4

Fornaciari, P.A., A-7

Frontera, M., A-14, A-15, A-17

G

Gaglioti, D., A-11

Galrito, M., A-14

Gambino, A., A-17

Garrone, M., A-17

Ghidini, G., A-6, A-14, A-16, A-17, A-18, A-20, A-21

Giordano, L., A-12

Giovannacci, I., A-6, A-15, A-17, A-18, A-19, A-21

Got, S.-R., A-4

Grajales, M., A-7

Graniero, F., A-11, A-18

Grecolini, M.E., A-7

Grosko, K.N., A-22

Grzech-Leśniak, K., A-9

\section{H}

Hakimiha, N., A-7

Hernán, G., A-6

Heysselaer, D., A-8, A-10

Houeis, S., A-8

$\mathbf{J}$

Jedlinski, M., A-8

Juvino, A., A-18

Juvino, A.C., A-8, A-13, A-21, A-22

$\mathbf{K}$

Karova, E., A-16

Kuchar, N.G., A-8, A-13, A-18, A-21
$\mathbf{L}$

Landi, M., A-19, A-20

Lietz-Kijak, D., A-8

Luca, G., A-12

Lucaciu, O., A-5

M

Magnoni, C., A-6, A-17, A-18, A-21

Manaheji, H., A-7

Manfredi, M., A-16, A-17

Manzoli, C., A-15, A-19

Marino, S., A-9, A-20

Matamba Kalombo, P., A-10

Meleti, M., A-6, A-14, A-16, A-18, A-19, A-20, A-21

Minardi, M., A-14, A-17

Misoaga, L., A-10

Mohsen, A., A-3, A-9

Mohsen, M., A-16

Moldoveanu, L.E., A-20

Monteiro, L., A-9, A-14, A-22

Montori, A., A-9, A-18

Mori, D., A-6

Moslemi, N., A-7

$\mathbf{N}$

Nahas, P., A-8

Nammour, S., A-6, A-8, A-10

Namour, A., A-10

Namour, M., A-10

Nathalia, Z., A-13

Nielsen, G K., A-13, A-18, A-21

$\mathbf{P}$

Pacheco, J., A-14, A-22

Palaia, G., A-9, A-10, A-18, A-20, A-22

Pedrazzi, G., A-18

Peremans, A., A-10

Pérez-Rodríguez, M.J., A-10

Pergolini, D., A-10, A-18, A-20, A-22

Pezzi, M.E., A-20

Pistoia, S., A-15

Podda, G.M., A-16, A-20

Porrini, M., A-4, A-9, A-15, A-19, A-20

$\mathbf{R}$

Rabelo, T., A-18

Rabelo, T.F., A-8, A-13, A-21

Raviola, E., A-12

Rebecchi, S., A-21

Reggiani, C., A-6, A-17, A-18, A-21

Renzi, F., A-22

Resende, T., A-22

Rey, G., A-11 
Rey, L., A-15, A-19

Rocchetti, F., A-3, A-9, A-11, A-20

Romagnoli, E., A-15

Romeo, U., A-9, A-10, A-11, A-22

Rompen, E., A-10

Rossi, M., A-4, A-9, A-20

\section{S}

Sala, A., A-18

Sala, R., A-6, A-18

Salazar, F., A-14, A-22

Setti, G., A-18

Sfondrini, M.F., A-14

Silva, R., A-22

Sonia, M., A-15

Soritau, O., A-5

Spadari, F., A-4, A-9, A-15, A-19, A-20

Stübinger, S., A-11
$\mathbf{T}$

Tenore, G., A-3, A-9, A-11, A-16, A-18, A-20

Thais Freitas, R., A-22

Todea, C., A-11, A-12

Truzzi, A., A-17

Tudose, A.D., A-12

Turatti, G., A-12

$\mathbf{U}$

Uhryn, M., A-4

Umberto, R., A-3

V

Valamvanos, K., A-12

Valentini, V., A-3
Valmaseda-Castellón, E., A-4

Veneri, F., A-14, A-19, A-20

Vescovi, P., A-6, A-14, A-15, A-16, A-17, A-18, A-19, A-20, A-21

Vigliaroli, E., A-15

Visca, P., A-22

Vitale, M.C., A-13, A-14

$\mathbf{Z}$

Zamataro, C.B., A-8, A-13, A-18, A-21

Zanini, N., A-8, A-13, A-18, A-21, A-22

Zeinoun, T., A-8, A-10, A-13

Zezell, D., A-8, A-22

Zezell, D.M., A-13, A-21

Zimbalatti, A., A-11 